\title{
Light quark masses in quenched QCD with exact chiral symmetry
}

\author{
Ting-Wai Chiu, Tung-Han Hsieh \\ Department of Physics, National Taiwan University, Taipei 106, Taiwan \\ Received 8 May 2002; accepted 23 May 2002 \\ Editor: M. Cvetič
}

\begin{abstract}
The parameters in the pseudoscalar meson mass formula in quenched chiral perturbation theory to one-loop order are determined by quenched lattice QCD with overlap Dirac operator, and from which the light quark masses are determined with the experimental inputs of pion and kaon masses, and the pion decay constant. Our results are $m_{u, d}=5.3 \pm 0.3 \mathrm{MeV}$, and $m_{s}=115 \pm 8 \mathrm{MeV}$, in the $\overline{\mathrm{MS}}$ scheme at scale $\mu=2 \mathrm{GeV}$. ( 2002 Elsevier Science B.V. All rights reserved.
\end{abstract}

PACS: 11.15.Ha; 11.30.Rd; 12.38.Gc

Keywords: Chiral perturbation theory; Chiral symmetry; Lattice QCD; Overlap Dirac quark

In the standard model, the quark masses are fundamental parameters which have to be determined from high energy experiments. However, they cannot be measured directly since quarks are confined inside hardrons, unlike an isolated electron whose mass and charge both can be measured directly from its responses in electric and magnetic fields. Therefore, the quark masses can only be determined by comparing a theoretical calculation of physical observables (e.g., hadron masses) with the experimental values. Evidently, for any field theoretic calculation, the quark masses depend on the regularization, as well as the renormalization scheme and scale.

One of the objectives of lattice QCD is to compute the hadron masses non-perturbatively from the first principle, and from which the quark masses are determined. However, the performance of the present

E-mail address: twchiu@phys.ntu.edu.tw (T.-W. Chiu). generation of computers is still quite remote from what is required for computing the light hadron masses at the physical (e.g., $m_{\pi} \simeq 140 \mathrm{MeV}$ ) scale, on a lattice with enough sites in each direction such that the discretization errors as well as the finite volume effects are both negligible comparing to the statistical ones. Nevertheless, even with lattices of moderate sizes, lattice QCD can determine the values of the parameters in the hadron mass formulas of the (quenched) chiral perturbation theory. Then one can use these formulas to evaluate the hadron masses at the physical scale, as well as to determine the quark masses.

In quenched chiral perturbation theory [1,2], the pion and kaon masses to one-loop order read as

$$
\begin{aligned}
& m_{\pi}^{2}=C m\left\{1-\delta\left[\ln \left(\frac{C m}{\Lambda_{\chi}^{2}}\right)+1\right]\right\}+B m^{2}, \\
& \frac{m_{K}^{2}}{m_{\pi}^{2}}=\frac{m+m_{s}}{2 m}\left\{1+\delta\left[1-\frac{m}{m_{s}-m} \ln \left(\frac{m_{s}}{m}\right)\right]\right\},
\end{aligned}
$$


where $m$ denotes $u$ and $d$ quark bare masses in the isospin limit $\left(m_{u}=m_{d} \equiv m\right), m_{s}$ the $s$ quark bare mass, $\Lambda_{\chi}=2 \sqrt{2} \pi f_{\pi}\left(f_{\pi} \simeq 132 \mathrm{MeV}\right)$ the chiral cutoff, $\delta$ the coefficient of the quenched chiral logarithm, and $C$ and $B$ are parameters. Similar to the Gell-Mann-Oakes-Renner relation,

$m_{\pi}^{2}=\frac{4 m\langle\bar{\psi} \psi\rangle}{f_{\pi}^{2}}$

the condensates to one-loop order are

$$
\begin{aligned}
& \langle\bar{u} u\rangle=\frac{f_{\pi}^{2} C}{4}\left\{1-\delta\left[\ln \left(\frac{C m}{\Lambda_{\chi}^{2}}\right)+1\right]+\frac{B m}{C}\right\}, \\
& \frac{\langle\bar{s} s\rangle}{\langle\bar{u} u\rangle}=\frac{m}{m_{s}}\left(\frac{2 m_{K}^{2}}{m_{\pi}^{2}}-1\right),
\end{aligned}
$$

where the parameter $C$ is related to the $u$ quark condensate at the zeroth order

$C=\frac{4\langle\bar{u} u\rangle_{0}}{f_{\pi}^{2}}$.

Note that the condensate $\langle\bar{u} u\rangle$ diverges logarithmically as the bare quark mass $m \rightarrow 0$, a pathology of quenched approximation. Thus the chiral symmetry breaking in QCD can only be properly addressed with dynamical quarks.

With experimental values of pion and kaon masses as inputs to (1) and (2), one can determine the ratio of light quark bare masses $m_{s} / m$, but the absolute scale cannot be fixed by quenched chiral perturbation theory. At this point, lattice QCD plays the important role to fix the values of parameters $C, B$ and $\delta$, by measuring the pion mass versus the bare quark mass. Then the light quark masses $m$ and $m_{s}$ can be determined with experimental inputs of $m_{\pi}, f_{\pi}$ (to fix the lattice spacing $a$ ), and $m_{K}$.

Recently, we have determined the parameters $C$, $B$ and $\delta$ [3] in lattice QCD with overlap Dirac operator [4,5]. For 100 gauge configurations generated with the Wilson gauge action at $\beta=5.8$ on the $8^{3} \times 24$ lattice, we computed quenched quark propagators for 12 bare quark masses. The pion decay constant $\left[f_{\pi} a=\right.$ $0.0984(3)]$ is extracted from the pion propagator, and from which the lattice spacing is determined to be

$a=0.147(1) \mathrm{fm}$.

By fitting (1) to our data of $\left(m_{\pi} a\right)^{2}$, we obtained

$\delta=0.2034(140)$,
$C a=1.1932(182)$,

$B=1.1518(556)$.

Now inserting (8) and experimental values of meson masses $\left(m_{K}=495 \mathrm{MeV}\right.$ and $\left.m_{\pi}=135 \mathrm{MeV}\right)$ into Eq. (2), we obtain the quark mass ratio

$\frac{m_{s}}{m}=21.92(23)$,

comparing with the ratio at the zeroth order $(\delta=0)$

$\left(\frac{m_{s}}{m}\right)_{0}=25.88$.

From (1), with values of $a, \delta, C a$ and $B$ in (7)-(10), and $m_{\pi}=135 \mathrm{MeV}$, we obtain

$m=6.3 \pm 0.3 \mathrm{MeV}$,

which is inserted into (11) to yield

$m_{s}=138 \pm 8 \mathrm{MeV}$.

Here we do not intend to estimate the finite volume and discretization errors, in view of the exploratory nature of our present investigation which will be followed by a systematic study with larger volumes, smaller lattice spacings, and better statistics.

In order to transcribe above results (13), (14) to their corresponding values in the usual renormalization scheme $\overline{\mathrm{MS}}$ in high energy phenomenology, one needs to compute the lattice renormalization constant $Z_{m}=Z_{s}^{-1}$, where $Z_{s}$ is the renormalization constant for $\bar{\psi} \psi$. In general, $Z_{m}$ should be determined nonperturbatively. In this Letter, we use the one loop perturbation formula [6] $\left(m_{0}=1.30\right)$

$Z_{s}=1+\frac{g^{2}}{4 \pi^{2}}\left[\ln \left(a^{2} \mu^{2}\right)+6.7722\right]$

to obtain an estimate of $Z_{s}$. At $\beta=5.8, a=0.147$ (1) $\mathrm{fm}$, and $\mu=2 \mathrm{GeV}$, (15) gives $Z_{s}=1.19(1)$. Thus the quark masses in (13), (14) are transcribed to

$m_{u, d}^{\overline{\mathrm{MS}}}(2 \mathrm{GeV})=5.3 \pm 0.3 \mathrm{MeV}$,

$m_{s}^{\overline{\mathrm{MS}}}(2 \mathrm{GeV})=115 \pm 8 \mathrm{MeV}$.

It is expected that above values may become smaller if non-perturbative renormalization is performed, however, the decreases should be less than $10 \%$. Nevertheless, the light quark masses in (16), (17) are in good agreement with the current lattice world average [7]. 
We also note that in previous determinations $[8,9]$ of quark masses in quenched QCD with overlap Dirac operator, $\left(m_{u, d}+m_{s}\right)$ is obtained [8,9] with (1) and (2) at the zeroth order $(\delta=0, B=0)$; while in Ref. [10], $m_{u, d}$ is obtained via the axial Ward identity.

In summary, the parameters of hadron mass formulas in (quenched) chiral perturbation theory can be fixed by lattice QCD with overlap Dirac quarks. This provides a viable approach to the determination of (light) quark masses, as well as (light) hadron masses.

\section{Acknowledgements}

This work was supported in part by the National Science Council, ROC, under the grant number NSC90-2112-M002-021, and also in part by NCTS.

\section{References}

[1] S.R. Sharpe, Phys. Rev. D 46 (1992) 3146.

[2] C.W. Bernard, M.F. Golterman, Phys. Rev. D 46 (1992) 853.

[3] T.W. Chiu, T.H. Hsieh, hep-lat/0204009.

[4] H. Neuberger, Phys. Lett. B 417 (1998) 141.

[5] R. Narayanan, H. Neuberger, Nucl. Phys. B 443 (1995) 305.

[6] C. Alexandrou, E. Follana, H. Panagopoulos, E. Vicari, Nucl. Phys. B 580 (2000) 394.

[7] For recent review and references, see, for example, T. Kaneko, Nucl. Phys. (Proc. Suppl.) 106 (2002) 133;

V. Lubicz, Nucl. Phys. (Proc. Suppl.) 94 (2001) 116.

[8] L. Giusti, C. Hoelbling, C. Rebbi, Phys. Rev. D 64 (2001) 114508.

[9] P. Hernandez, K. Jansen, L. Lellouch, H. Wittig, Nucl. Phys. (Proc. Suppl.) 106 (2002) 766.

[10] S.J. Dong, F.X. Lee, K.F. Liu, J.B. Zhang, Phys. Rev. Lett. 85 (2000) 5051. 